\title{
ERRATUM
}

Jennifer R. Seal · Ned A. Porter

\section{Liquid chromatography coordination ion-spray mass spectrometry (LC-CIS-MS) of docosahexaenoate ester hydroperoxides}

Published online: 26 August 2004

(C) Springer-Verlag 2004

\section{Anal Bioanal Chem (2004) 378: 1007-1013}

We published a paper entitled "Liquid chromatography coordination ion-spray mass spectrometry (LC-CISMS) of docosahexaenoate ester hydroperoxides" in a recent issue of this journal (Anal Bioanal Chem (2004) 378: 1007-1013). It has since been brought to our attention that we regretfully failed to reference a previous paper describing the identification of methyl docosahexaenoate (Me-HDHE) alcohols by GC-MS, (VanRollins, Mike; Murphy, Robert C. "Autooxidation of docosahexaenoic acid: analysis of ten isomers of hydroxydocosahexaenoate," (1984) J Lipid Res 25: 507517). In this paper, the authors isolated HDHEs by reverse phase HPLC, converted them to their methyl esters, and purified them by normal phase HPLC. Once purified, they were silylated and catalytically hydrogenated then structurally identified by electron impact GC-MS. In our contribution, we reported a new method for HPLC analysis of intact DHA hydroperoxides, Coordination Ion-Spray Mass Spectrometry, and describe the identification of not only the methyl ester hydroperoxides but also the phospholipid and cholesterol ester hydroperoxides of DHA. The advantage of our mass spectrometric technique lies in the fact that no prior derivatization steps are necessary to identify the positional isomers of each hydroperoxide. We have no reason to dispute the elution order of the Me-HDHEs reported by Drs. VanRollins and Murphy. However, our mass spectrometric method unambiguously supports the elution order reported in our manuscript and the differences between the two are most likely due to differences in column and solvent composition. We sincerely regret not referencing Drs. VanRollins and Murphy's contribution as they lay important groundwork in this field of chemistry.

Jennifer R. Seal

Ned A. Porter

The online version of the original article can be found at http:// dx.doi.org/10.1007/s00216-003-2274-6

\footnotetext{
J. R. Seal · N. A. Porter $(\bowtie)$

Department of Chemistry, Center in Molecular Toxicology, Vanderbilt University, Nashville, TN 37235, USA

E-mail: n.porter@vanderbilt.edu
} 